\title{
A Numerical Investigation on a Hybrid- Parameterless Radial Basis Function Applied with a Meshless Method
}

\author{
T Moonsan', S Kaennakham 2,3*, N Chuathong ${ }^{4}$ \\ 1. Department of Mathematics, Faculty of Science, Udon \\ Thani Rajabhat University, Udon Thani, Thailand \\ 2. School of Mathematics, Institute of Science, Suranaree \\ University of Technology, Nakhon Ratchasima, Thailand \\ 3. Centre of Excellence in Mathematics, Bangkok, Thailand \\ 4. Faculty of Science, Energy and Environment, King \\ Mongkut's University of Technology North Bangkok, \\ Rayong, Thailand
}

\begin{abstract}
A Hybrid RBF has recently been proposed and tested with some scattered data interpolation problems and the results have appeared promising whereas the appearance of the shape parameter remains a difficulty when deploying. This work, therefore, focuses on three objectives; firstly, it is aimed to extend the use of the newly proposed-RBF to application of RBFcollocation method. Secondly, realizing the burden attributed to the lack of information on choosing an optimum shape parameter, the hybrid RBF is then modified where the shape parameter is no longer included. Thirdly, it is to investigate its application/implementation towards solving PDEs particularly those in both linear and non-linear form. It has been found in this work that the new RBF of this HyBrid form with no parameter can well be a good candidate and truly deserves further study with more complex problems.
\end{abstract}

\section{INTRODUCTION}

Appearing as an alternative numerical tool for numerically solving Partial Differential Equations (PDEs), the so-called 'meshless' or 'meshfree' method has been receiving a great amount of attention from both scientists and engineers. Unlike those traditional numerical methods; Finite Volume (FV), Finite Element (FE), or Finite Difference (FD), meshless method doesn't depend on any connectivity amongst nodes making it free of the burden of mesh generating process. A collocation method based on Radial Basis Functions (RBF) is considered as truly meshfree scheme where no mesh is needed at all. The idea of implementing the collocation method to solving problems in differential equations was first attempted by Kansa [1], this is why the method of collocation based on RBF is sometimes called 'Kansa's method'. The main advantages of RBF-based methods for solving PDEs lie on their simplicity, their applicability to various PDEs, and their effectiveness in dealing with high dimensional problems with complex geometry domains [2]. 
Kansa's method is consist of several important components and one of which is the choice of the RBF used itself. Despite of the large number of researches devoting to finding an optimal RBF [3-6], it has been accepted that the most suitable RBF depends very much on the nature of the problem (with its conditions) at hand. Our previous study [7] focused on the effectiveness of several well-known and mostly-used RBFs for nonlinear class of PDE. In this work, it has been found that while Gaussian-RBF produces the reasonably worst results' quality, the so-called Cubic Matérn RBF type is found to yield the best results' quality for all test cases.

Recently, a new RBF which is a combination of Gaussian and Cubic type, has been invented and proposed by Mishra et. al. [8]. In their work, an attempt to alleviate the problem of ill-condition normally encountered when using Kansa' method is made. The proposed combined-RBF was applied in several benchmark scattered-data interpolation problems; 1D, $2 \mathrm{D}$ and $3 \mathrm{D}$. This immediately prompts the question of applying this newly invented RBF to problems of solving PDE, particularly non-linear ones via. a collocation-based meshfree method.

While desirable aspects of the Hybrid-RBF were discovered, it is to mention that the choice of suitable shape parameter is not straightforward meaning that it is often selected in a 'adhoc' manner. To alleviate or even eliminate this uncertainty, it is one of this investigation's aim that a newly modified version of this Hybrid-RBF is to be proposed and tested.

\section{GLOBAL COLLOCATION-BASED METHOD}

Radial Basis Functions (RBF), $\varphi$, are commonly found as multivariate functions whose values are dependent only on the distance from the origin. This means that $\varphi(x)=\varphi(r) \in \mathbb{R}$ with $x \in \mathbb{R}^{n}$ and $r \in \mathbb{R}$, or, in other words, on the distance from a point of a given $\operatorname{set}\left\{x_{j}\right\}$, and $\varphi\left(x-x_{j}\right)=\varphi\left(r_{j}\right) \in \mathbb{R}$.Here, $r_{j}$ is the Euclidean distance defined as follows.

$$
r_{i j}=\left\|x_{i}-x_{j}\right\|_{2}=\left[\left(x_{i}^{(1)}-x_{j}^{(1)}\right)^{2}+\left(x_{i}^{(2)}-x_{j}^{(2)}\right)^{2}+\ldots+\left(x_{i}^{(n)}-x_{j}^{(n)}\right)^{2}\right]^{1 / 2}
$$

And any function $\varphi$ satisfying $\varphi(x)=\varphi\left(\|x\|_{2}\right)$ is a radial function. This work defines $\varphi(x)=\varphi\left(\|x\|_{2}\right)$ throughout. $\sqrt[2]{w d}$

For the methodology of RBF-collocation for numerically solving PDEs, it begins with considering a linear elliptic partial differential equation with boundary conditions, where $g(x)$ and $f(x)$ are known. We seek $u(x)$ that satisfies, [1].

$$
\begin{gathered}
\operatorname{Lu}(x)=f(x), \quad x \text { in } \Omega \\
M u(x)=g(x), \quad x \text { in } \partial \Omega
\end{gathered}
$$

where $\Omega \in \mathbb{R}^{d}, \partial \Omega$ denotes the boundary of domain $\Omega, L$ and $M$ are the linear elliptic partial differential operators and operating on the domain $\Omega$ and boundary domain $\partial \Omega$, respectively. For Kansa's method, it represents the approximate solution by the interpolation, using an RBF interpolation as the following; 


$$
\tilde{u}(x)=\sum_{j=1}^{N} c_{j} \varphi\left(\left\|x-x_{j}\right\|\right)
$$

where $\|$. $\|$ denote the Euclidean norm in $\mathbb{R}^{2}$. We can see that $N$ unknowns of $c_{j}$. Substituting $\tilde{u}(x)$ into (1) and (2), we obtain the system of equations as follows.

$$
L\left(\sum_{j=1}^{N_{I}} c_{j} \varphi\left\|x-x_{j}\right\|\right)=\sum_{j=1}^{N_{I}} c_{j} L \varphi\left(\left\|x-x_{j}\right\|\right)=f\left(x_{i}\right),
$$

for $=1, \ldots, N_{I}$, and

$$
M\left(\sum_{j=N_{i}+1}^{N} c_{j} \varphi\left\|x-x_{j}\right\|\right)=\sum_{j=N_{i}+1}^{N} c_{j} M \varphi\left(\left\|x-x_{j}\right\|\right)=g\left(x_{i}\right),
$$

where $=N_{I}+1, \ldots, N$.

Above equations, we choose $N$ collocation points on both domain $\Omega$ and boundary domain $\partial \Omega$, and divide it into $N_{I}$ interior points and $N_{B}$ boundary points $\left(N=N_{I}+N_{B}\right)$. Let $X=$ $\left\{x_{1}, x_{2}, \ldots, x_{N}\right\}$ denotes the set of collocation points, $I=\left\{I_{1}, \ldots, I_{N_{I}}\right\}$ denotes the set of interior points and $B=\left\{B_{1}, \ldots, B_{N_{B}}\right\}$ the set of boundary points. The centers $x_{j}$ used in equation (5) and equation (6) are chosen as collocation points. The previous substituting yields a system of linear algebraic equations which can be solved for seeking coefficient $c$ by rewriting equation (5) and equation (6) in matrix form as;

$$
A c=F
$$

where $A=\left[A_{L}, A_{M}\right]^{T}$ and $F=\left[f\left(x_{i}\right), g\left(x_{i}\right)\right]^{T}$ with the following submatrices.

$$
\begin{aligned}
& \left(A_{L}\right)_{i j}=L \varphi\left(\left\|x_{i}-x_{j}\right\|\right), x_{i} \in I, x_{j} \in X, i=1,2, \ldots, N_{I}, j=1,2, \ldots, N, \\
& \left(A_{M}\right)_{i j}=M \varphi\left(\left\|x_{i}-x_{j}\right\|\right), x_{i} \in B, x_{j} \in X, i=N_{I}+1, \ldots, N, j=1,2, \ldots, N, \\
& f\left(x_{i}\right) ; x_{i} \in I, \quad i=1,2, \ldots, N_{I}, \\
& g\left(x_{i}\right) ; x_{i} \in B, \quad i=N_{I}+1, \ldots, N .
\end{aligned}
$$

The matrix $A$ in equation (7) is non-singular [1] and therefore, the coefficient $c^{\prime} s$ are computed from the following system;

$$
c=A^{-1} F
$$


The matrix $c$ is substituted into equation (4) and the approximate solution of $\tilde{u}(x)$ can be determined by

$$
\tilde{u}(x)=\sum_{j=1}^{N} c_{j} \varphi\left(\left\|x-x_{j}\right\|\right)
$$

\section{THE PROPOSED RADIAL BASIS FUNCTION}

As proposed and tested out with a series of numerical tests for 1D, 2D and 3D problems as well as synthetic and real geophysical data by Mishra et. al. [8], this work is now being expanded to another challenging problem of solving PDEs. The Hybrid RBF is of the following form;

$$
\varphi_{H y b}\left(\left\|x-x_{j}\right\|\right)=\exp \left(-\left(\rho\left\|x-x_{j}\right\|\right)^{2}\right)+\left\|x-x_{j}\right\|^{3}
$$

This is referred to, hereafter, as 'HyB'. The first component $\exp \left(-\left(\rho\left\|x-x_{j}\right\|\right)^{2}\right)$ is Gaussian part which is shape parameter dependent while the Cubic part, $\left\|x-x_{j}\right\|^{3}$, does not contain any shape parameter term. This combination of RBF makes it 'Gaussian radial basis function with small cubic doping' for large shape parameter whereas for small shape parameter, the cubic term dominates the kernel making it 'Cubic radial basis function with small Gaussian doping'. It is noted, however, that the first part can still be controlled and strongly affected by the choice of parameter which is not straightforward in practical use. To provide an example of this burden, one of our previous studies [9] is now being visited and FIG.1. depicts the nonlinear relation of solution accuracy and the change of shape parameter.

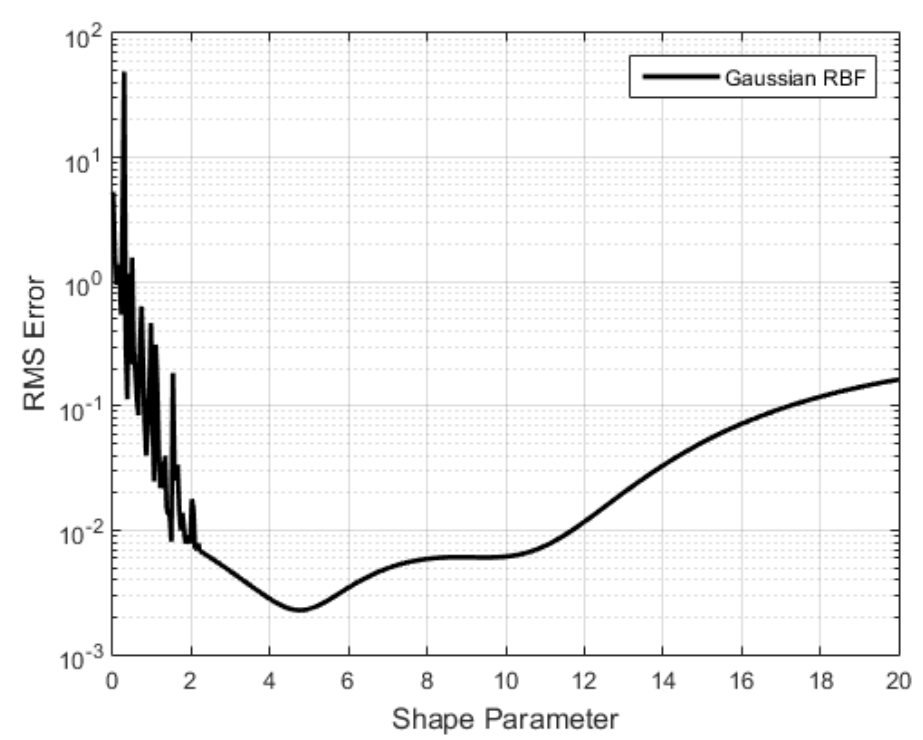

FIG. 1. Root Mean Square Error (RMSE) in the interpolation problem of the wellknown Franke-type function with Gaussian-RBF, obtained at various shape parameters when computed using 149 interpolation nodes (see [9]). 
For this reason, formula (10) is now modified further in order to completely eliminate the burden of choosing suitable parameter. It, instead, is designed to rely only on the distance between centers alone where an extra weighting function, $\xi$ locally defined, is added. The proposed modified form is defined as follows.

$$
\varphi_{M d H y b}^{i j}\left(\left\|x_{i}-x_{j}\right\|_{2}\right)=\left(1-\xi_{i j}\right) \exp \left(-\left(\left\|x_{i}-x_{j}\right\|_{2}\right)^{2}\right)+\xi_{i j}\left(\left\|x_{i}-x_{j}\right\|_{2}\right)^{3}
$$

where the new locally-auto adaptive parameter is proposed by.

$$
\xi_{i j}=\frac{\left\|x_{i}-x_{j}\right\|_{2}}{\max _{1 \leq i, j \leq N}\left\|x_{i}-x_{j}\right\|_{2}}
$$

and this form shall be named as 'MdHyB' throughout the paper.

\section{THE METHOD'S IMPLEMENTATION}

\subsection{Space Discretization for Linear Terms}

Consider the governing partial differential equation of convection-diffusion problems expressed as [10-11];

$$
\frac{\partial u}{\partial t}-\alpha\left(\frac{\partial^{2} u}{\partial x^{2}}+\frac{\partial^{2} u}{\partial y^{2}}\right)+\beta\left(\frac{\partial u}{\partial x}+\frac{\partial u}{\partial y}\right)=R
$$

where $\alpha, \beta$ are the diffusive and convective term, respectively and $R$ describes source or sinks of the quantity $\alpha$. In steady state case, the convection-diffusion equation is reduced to the following steady state form;

$$
-\alpha\left(\frac{\partial^{2} u}{\partial x^{2}}+\frac{\partial^{2} u}{\partial y^{2}}\right)+\beta\left(\frac{\partial u}{\partial x}+\frac{\partial u}{\partial y}\right)=R
$$

$\operatorname{For}(x, y) \in \Omega \subset \mathbb{R}^{d}$. In this practice, its boundary condition is of Dirichlet type as follows:

$$
u(x, y)=0
$$

on $\partial \Omega$.

In order to implement the Kansa meshless procedure; equations (4-6) are applied to equation (14), it is necessary to solve the following linear system.

$$
\sum_{j=1}^{N} c_{j}\left[-\alpha\left(\frac{\partial^{2} \varphi\left(\left\|x_{i}-x_{j}\right\|\right)}{\partial x^{2}}+\frac{\partial^{2} \varphi\left(\left\|x_{i}-x_{j}\right\|\right)}{\partial y^{2}}\right)+\beta\left(\frac{\partial \varphi\left(\left\|x_{i}-x_{j}\right\|\right)}{\partial x}+\frac{\partial \varphi\left(\left\|x_{i}-x_{j}\right\|\right)}{\partial y}\right)\right]=R
$$

for $i=1, \ldots, N_{I}$ and;

$$
\sum_{j=1}^{N} c_{j} \varphi\left(x_{i}-x_{j}\right)=0
$$


for $i=N_{I}+1, \ldots, N$. This system can be written in the form of;

$$
\sum_{j=1}^{N} c_{j}\left[-\alpha\left[\nabla^{2} \varphi\right]_{i j}+\beta[\nabla \varphi]_{i j}\right]=R,
$$

for and $i=1, \ldots, N_{I}$

$$
\sum_{j=1}^{N} c_{j} \varphi_{i j}=0
$$

where $i=N_{I}+1, \ldots, N$.

This system can be generated in matrix form as equation (7), then, the approximate solutions then can be obtained by substituting the coefficients obtained by solving the above matrix form, in equation (8).

\subsection{Dealing with Non-linear Terms}

The computation process starts with considering the nonlinear PDEs of the form

$$
L u=f
$$

with the following algorithm;

1) Create the collocation point sets $X \subset \Omega$ and start with an initial guess $u_{0}$.

2) For $k=1,2, \ldots, K$ the linearized problem $\Lambda_{u_{k-1}} v=f-L u_{k-1}$ on $X$

a) Perform the Newton update

$$
\tilde{v}=v
$$

b) Update the previous approximation

$$
u_{k}=u_{k-1}+\tilde{v}
$$

In this algorithm $\Lambda_{u_{k-1}}$ is the linearization of the nonlinear differential operator $L$ at $u_{k-1}$. Here we provide an example in order to elaborate the algorithm described above.

Considering the nonlinear PDE of the form2;

$$
\begin{gathered}
-E^{2} \nabla^{2} u-u+u^{3}=f \text { in } \Omega=(0,1) \times(0,1) \\
u=0 \text { on } \partial \Omega
\end{gathered}
$$

On the right hand side, $f$ is chosen so that equation (21) has an analytic solution of the form.

$$
u(x, y)=\psi(x) \psi(y)
$$


with $\psi(t)=1+e^{-1 / E}-e^{t / E}-e^{(t-1) / E},(x, y)$ denotes the Cartesian coordinates of $x \in \mathbb{R}^{2}$, and the parameter $\varepsilon$ determines the size of the boundary layers near the edges of the domain $\Omega$. We use a value of $E=0.1$. For this model problem the linearization of $\Lambda_{u_{k-1}}$ is given by;

$$
\Lambda_{u_{k-1}} v=-E^{2} \nabla^{2} v+\left(3 u_{k-1}^{2}-1\right) v
$$

and therefore the equation to be solved in step 2a) of the algorithm is of the form

$$
-E^{2} \nabla^{2} v+\left(3 u_{k-1}^{2}-1\right) v=f+E^{2} \nabla^{2} u_{k-1}+u_{k-1}-u_{k-1}^{3}
$$

When perform step 2a), it is necessary to solve the following linear system, arising from the nonsymmetrical collocation procedure, then the above equation becomes.

$$
\sum_{j=1}^{n^{(k)}} c_{j}^{(k)} \Lambda_{u_{k-1}}\left[\varphi\left(\left\|x-x_{j}^{(k)}\right\|\right)\right]_{x=x_{j}^{(k)}}=f+\Lambda u_{k-1}\left(x_{i}^{(k)}\right)
$$

where $i=1, \ldots, n_{I}^{(k)}$

$$
\sum_{j=1}^{n} c_{j}^{(k)}\left[\varphi\left(\left\|x_{i}^{(k)}-x_{j}^{(k)}\right\|\right)\right]=0
$$

where $i=n_{I}^{(k)}+1, \ldots, n^{(k)}$

By using equations (24-27), the above then becomes;

$$
\sum_{j=1}^{n} c_{j}\left[-E^{2}\left[\nabla^{2} \varphi\right]_{i j}+\left(3 u_{i}^{2}-1\right) \varphi_{i j}\right]=f+E^{2}\left[\tilde{\nabla}^{2} u\right]_{i}+u_{i}-u_{i}^{3}
$$

where $i=1, \ldots, n_{I}$ and

$$
\sum_{j=1}^{n} c_{j} \varphi_{i j}=0
$$

where $i=n_{I}+1, \ldots, n_{I}+n_{B}=n$, and for transparency, the index $k^{\prime} s$ are being omitted on the quantities $n, n_{I}, n_{B}$ and $c_{j}$.

The Newton update $v \%=v$ used in step $2 \mathrm{~b}$ ) of the algorithm is then given by;

$$
v(x)=\sum_{j=1}^{n^{(k)}} c_{j}^{(k)} \varphi\left(\left\|x-x_{j}^{(k)}\right\|\right)
$$




\subsection{Time-discretization}

In this numerical experiment the explicit fourth-order Range-Kutta (RK4) method is utilized to solve the problems. We assume with following the $u\left(x, t_{n}\right)=u^{n}, x \in \mathbb{R}^{2}$

$$
\begin{aligned}
& k_{1}=\Delta t F\left(u^{n}, t^{n}\right), \\
& k_{2}=\Delta t F\left(u^{n}+\frac{k_{1}}{2}, t^{n}+\frac{\Delta t}{2}\right), \\
& k_{3}=\Delta t F\left(u^{n}+\frac{k_{2}}{2}, t^{n}+\frac{\Delta t}{2}\right), \\
& k_{4}=\Delta t F\left(u^{n}+k_{3}, t^{n}+\Delta t\right)
\end{aligned}
$$

Then the updated-value of the solution at the new time step is calculated by the following expression.

$$
u^{n+1}=u^{n}+\frac{1}{6}\left(k_{1}+2 k_{2}+2 k_{3}+k_{4}\right)
$$

The next section, the whole process of numerical computing is implemented for solving linear and nonlinear partial differential equations and for this, three problems are selected and tested. All solutions obtained from this investigation are validated against with their corresponding exact solutions and also those obtained from literature when possible.

\section{NUMERICAL RESULTS AND GENERAL DISCUSSION}

In order to compare the effectiveness of the proposed RBF, some popular choices of RBFs have been included in the investigation. For the sake of clarification, some RBFs involved are listed once more time as follows, [12-14].

- Gaussian (GS): $\varphi(r)=\exp \left(-(\rho r)^{2}\right)$

- Inverse Multiquadric (IMQ): $\varphi(r)=1 / \sqrt{1+(\rho r)^{2}}$

- Multiquadric (MQ): $\varphi(r)=\sqrt{1+(\rho r)^{2}}$

- Inverse quadratic (IQ): $\varphi(r)=1 /\left[1+(\rho r)^{2}\right]$

- Wendland $C^{6}(\mathrm{WL}): \varphi(r)=(1-\rho r)^{8}\left(32(\rho r)^{3}+25(\rho r)^{2}+8 \rho r+1\right)$

- Cubic Matérn (CU): $\varphi(r)=e^{-\rho r}\left(15+15 \rho r+6(\rho r)^{2}+(\rho r)^{3}\right)$

where, $\rho$ is called shape parameter, determined by the user.

The results obtained thorough this work is verified against their exact ones and those found in literature, if available. All simulations shown were run on Intel(R) Core(TM) i7-2620M CPU @ 2.70 GHz and RAM 4.00 GB. 


\subsection{Experiment 1}

The first test case is the nonlinear PDE given in Fasshauer [15], defined on a square $\operatorname{domain}(x, y) \in(0,1) \times(0,1)$. The governing equation is as follows.

$$
-\varepsilon^{2} \nabla^{2} u-u+u^{3}=f
$$

with the boundary condition $u=0$ on $\partial \Omega$ and the right hand side of the equation is chosen from the analytical solution of form;

$$
u(x, y)=\psi(x) \psi(y)
$$

with

$$
\psi(t)=1+e^{-1 / \varepsilon}-e^{-t / \varepsilon}-e^{(t-1) / \varepsilon}
$$

and $(x, y)$ denotes the Cartesian coordinate of $x \in \mathbb{R}^{2}$.

The solutions are listed in Table 1 where a corresponding numerical study is contained. The best shape parameter used for 'HyB' is found to be in $\rho \in(1.2,2.3)$.

Table. 1 Solutions comparison of solutions obtained in this work ( $\varepsilon=1.00)$ and both alternative numerical work, WL-RBF used in Chuatong et al. [7], and the exact ones.

\begin{tabular}{lllll}
\hline Point & Chuatong et al. [7] & HyB [8] & MdHyB & Exact \\
\hline$(0.2,0.2)$ & 0.751694 & 0.84959432 & 0.7496651 & 0.747144 \\
$(0.2,0.6)$ & 0.848346 & 0.87731258 & 0.8412444 & 0.846440 \\
$(0.4,0.4)$ & 0.958317 & 0.92524858 & 0.9602457 & 0.958933 \\
$(0.4,0.6)$ & 0.958317 & 0.92524858 & 0.9820147 & 0.958933 \\
$(0.6,0.8)$ & 0.848346 & 0.87731258 & 0.8411786 & 0.846440 \\
$(0.8,0.4)$ & 0.848346 & 0.87731258 & 0.8327848 & 0.846440 \\
$(0.2,0.2)$ & 0.751694 & 0.84959432 & 0.7912117 & 0.747144 \\
$(0.2,0.4)$ & 0.848346 & 0.87731258 & 0.8600159 & 0.846440 \\
$(0.2,0.2)$ & 0.751694 & 0.84959432 & 0.7496651 & 0.747144 \\
\hline
\end{tabular}

\subsection{Experiment 2}

The following nonlinear equation as given in Linesawat [16] is studied. The governing equation is as follows.

$$
\Delta^{2} u=2 u^{3}
$$

defined in a domain $(1,5) \times(1,5)$ with the Dirichlet boundary condition $u=-1 / x$ on $\partial \Omega$. The analytical solution of the above problem is $u=-1 / x$. The results obtained are shown in 
Table 2 alongside with the alternation numerical study. FIG. 2. displays solution profiles produced by using the proposed RBF and by the exact solutions. The best shape parameter used for 'HyB' is found to be in $\rho \in(1.75,2.24)$.

Table. 2 Solutions comparison of those obtained in this work and both alternative numerical work, WL-RBF used in Linesawat [16], and the exact ones.

\begin{tabular}{lllll}
\hline Point & Linesawat [16] & HyB [8] & MdHyB & Exact \\
\hline$(2,2)$ & -0.5047 & -0.4751 & -0.5124 & -0.5000 \\
$(2,3)$ & -0.4993 & -0.4767 & -0.4804 & -0.5000 \\
$(3,3)$ & -0.3358 & -0.2008 & -0.3014 & -0.3333 \\
$(3,4)$ & -0.3290 & -0.2237 & -0.3022 & -0.3333 \\
$(4,4)$ & -0.2290 & -0.1545 & -0.2398 & -0.2500 \\
\hline
\end{tabular}

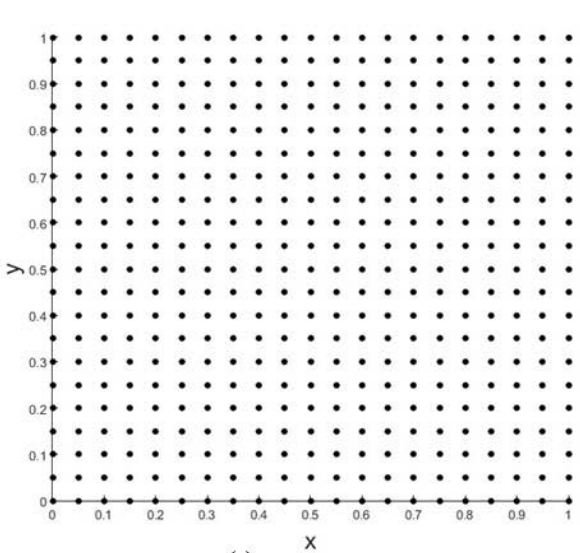

(a)

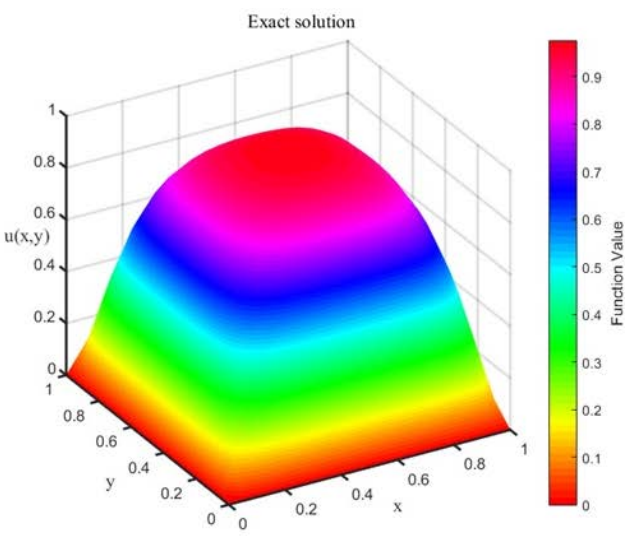

(b)

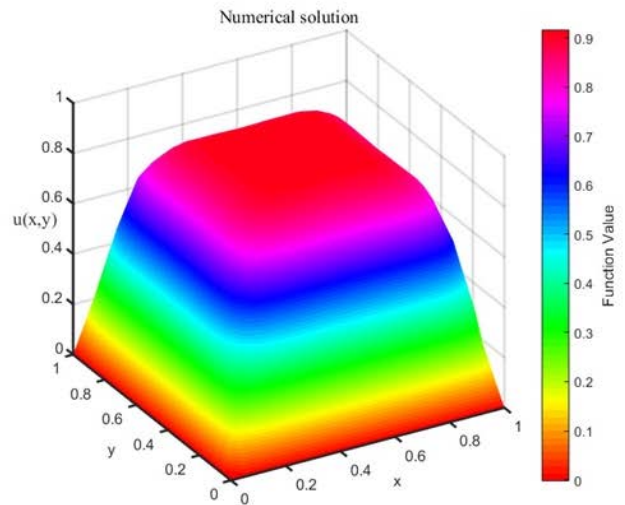

(c)

FIG. 2. Solution (a) Computation domain containing 441 uniformly-distributed nodes, (b) Exact solution profile, and (c) Numerical solution profile using Gaussian RBF with $\rho=10.00$. 


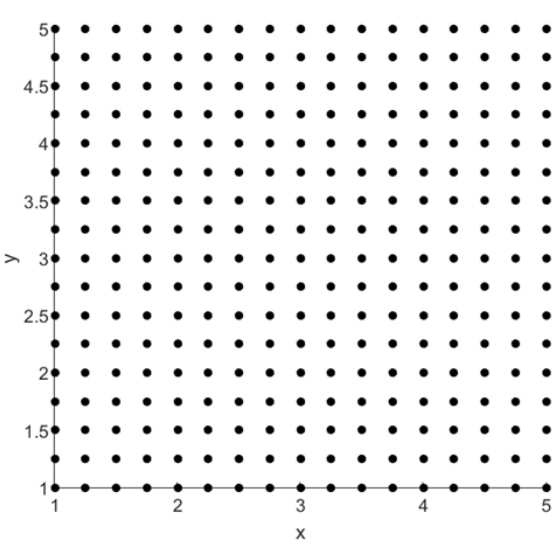

(a)

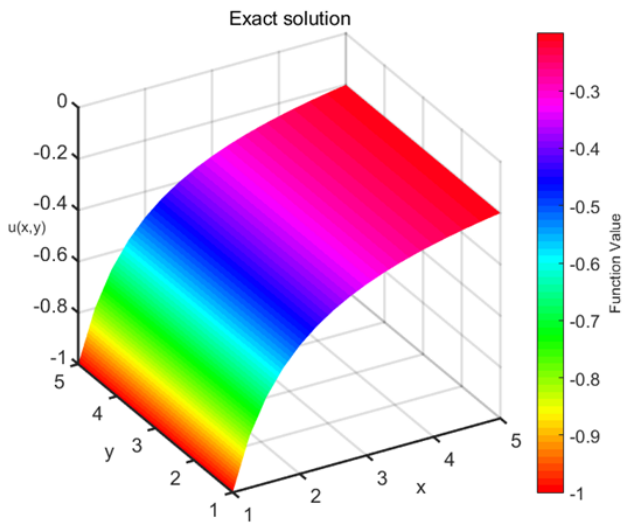

(b)

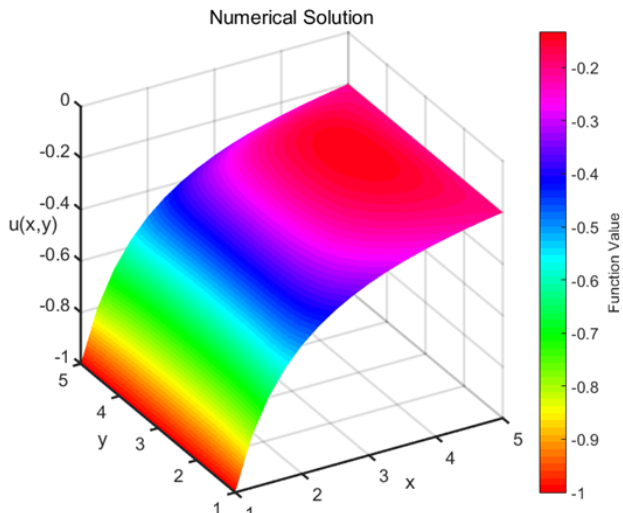

(c)

FIG. 3. Solution (a) Computation domain containing 441 uniformly-distributed nodes, (b) Exact solution profile, and (c) Numerical solution obtained from the proposed RBF(MdHyB).

\subsection{Experiment 3}

In this second case study, we focus now on the two-dimensional linear problem in timedependence state as given and studied by [17] The governing equation is as follows.

$$
u_{t}+u_{x}+u_{y}=\alpha\left(u_{x x}+u_{y y}\right)
$$

with initial condition $u(x, y, 0)=\sin (x+y)$ on $[0,2 \pi] \times[0,2 \pi]$ and periodic boundary conditions, where $\alpha=0.001$. The exact solution is as follows.

$$
u(x, y, t)=\exp (-2 t \pi) \sin (x+y-2 t)
$$


To deal with the time-derivative term appeared in equation (37), we employed the fourth order Runge-Kutta method (RK4). For solution validation for this problem, the root-meansquare error type defined blow is employed.

$$
L_{r m s}=\left(\frac{1}{N} \sum_{j=1}^{N}\left(\tilde{u}\left(x_{j}\right)-u\left(x_{j}\right)\right)^{2}\right)^{1 / 2}
$$

Table 3 provides $L_{r m s}$ produced by different types of RBFs with also different levels of node density. Some popular choices of RBFs listed in Section 5 are now under consideration. At least 4 levels of node density have been experimented on to show the sensitivity of the optimal shape parameter when the distance between nodes is changing. For example, in the case of the Multiquadric RBF (MQ), it can be seen from the table that during the first 3 levels of node density (8x8 to $24 \times 24$ ) the optimal shape discovered shows an increasing trend from $\rho_{\text {best }}=0.15$ to $\rho_{\text {best }}=2.55$. Nevertheless, the new rather surprising optimal shape then drops to $\rho_{\text {best }}=0.2$ when nodes are denser. This situation also appears to be the case for all types of RBFs contained in the same table confirming the difficulty of finding or pin-pointing the optimal value (if any) of the so-called 'shape-parameter'. Having a shape that can be selfadaptive, as a result, can be great of benefit where satisfactory result accuracy can still be expected and this all is clearly shown in Table 3.

Table. 3 Root-mean-square error $\left(L_{r m s}\right)$ obtained from each RBF type under investigation with their best value of shape parameter $\left(\rho_{\text {best }}\right)$; simulation at $t=0.5 \mathrm{~s}$, with $\Delta t=0.05$.

\begin{tabular}{|c|c|c|c|c|}
\hline $\mathbf{N}$ & & $8 \times 8$ & & $6 \times 16$ \\
\hline$\overline{\text { MQ }}$ & $2.05 \mathrm{E}-03$ & $\rho_{\text {best }}=0.15$ & 2.99E-03 & $\rho_{\text {best }}=0.74$ \\
\hline IMQ & $2.14 \mathrm{E}-02$ & $\rho_{\text {best }}=1$ & $3.02 \mathrm{E}-03$ & $\rho_{\text {best }}=2.25$ \\
\hline GS & $1.98 \mathrm{E}-01$ & $\rho_{\text {best }}=15$ & 8.23E-03 & $\rho_{\text {best }}=21.05$ \\
\hline IQ & 2.70E-02 & $\rho_{\text {best }}=25.22$ & $6.05 \mathrm{E}-03$ & $\rho_{\text {best }}=18$ \\
\hline WL & 2.11E-01 & $\rho_{\text {best }}=5$ & 2.05E-02 & $\rho_{\text {best }}=12.25$ \\
\hline НyВ [8] & 2.07E-02 & $\rho_{\text {best }}=8.2$ & $6.04 \mathrm{E}-02$ & $\rho_{\text {best }}=2.5$ \\
\hline МdHyB & $5.26 \mathrm{E}-02$ & & 2.30E-02 & \\
\hline $\mathbf{N}$ & & $24 \times 24$ & & $2 \times 32$ \\
\hline$\overline{\text { MQ }}$ & $1.54 \mathrm{E}-03$ & $\rho_{\text {best }}=2.55$ & $8.24 \mathrm{E}-04$ & $\rho_{\text {best }}=0.2$ \\
\hline IMQ & 3.18E-03 & $\rho_{\text {best }}=2$ & $3.12 \mathrm{E}-03$ & $\rho_{\text {best }}=2.55$ \\
\hline GS & 2.05E-02 & $\rho_{\text {best }}=11.2$ & $5.21 \mathrm{E}-03$ & $\rho_{\text {best }}=18.5$ \\
\hline IQ & 4.85E-03 & $\rho_{\text {best }}=15.2$ & $5.25 \mathrm{E}-03$ & $\rho_{\text {best }}=10.23$ \\
\hline WL & $1.74 \mathrm{E}-02$ & $\rho_{\text {best }}=21$ & 7.45E-03 & $\rho_{\text {best }}=25.5$ \\
\hline НyВ [8] & 4.28E-03 & $\rho_{\text {best }}=8$ & $1.25 \mathrm{E}-03$ & $\rho_{\text {best }}=15$ \\
\hline МdHyB & $1.22 \mathrm{E}-02$ & & $5.04 \mathrm{E}-03$ & \\
\hline
\end{tabular}



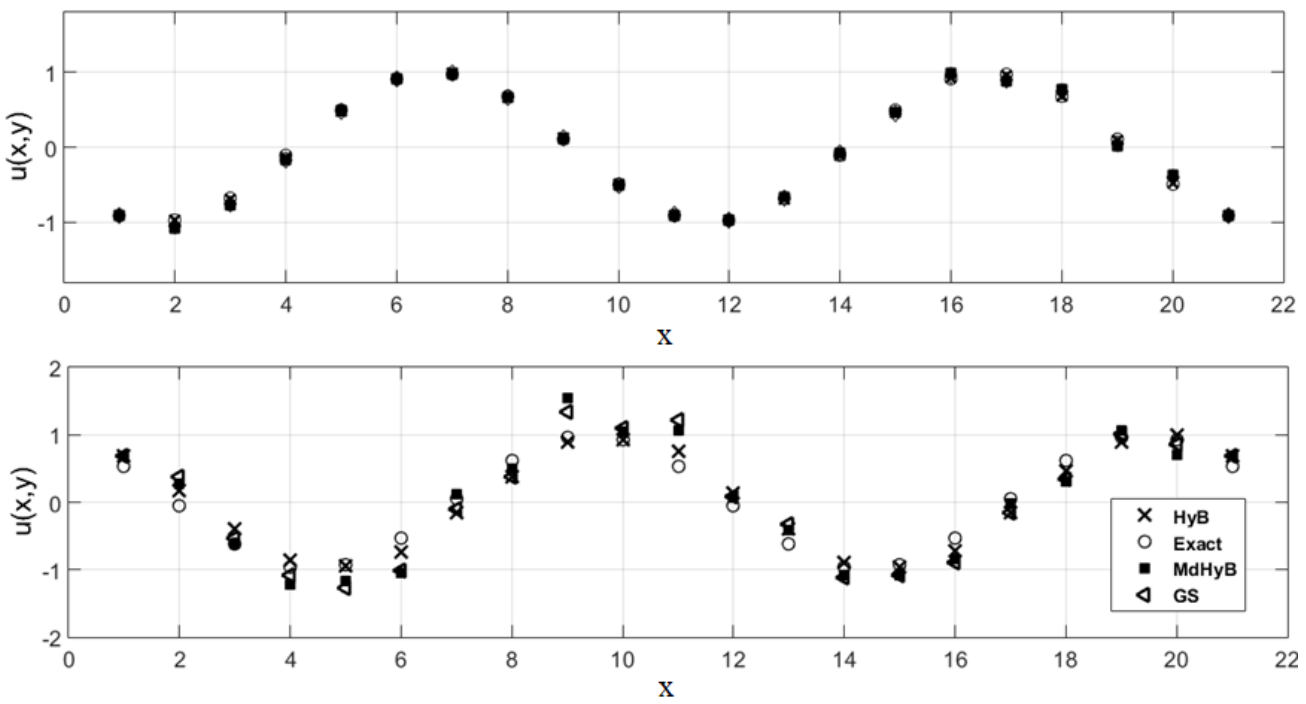

FIG. 4. Numerical solution measured along the straight line using 441 uniformlydistributed computation nodes; (above) at $t=1.0 \mathrm{~s}$, with $\Delta t=0.05$, and (below) at $t=$ $5.0 s$, with $\Delta t=0.1$.

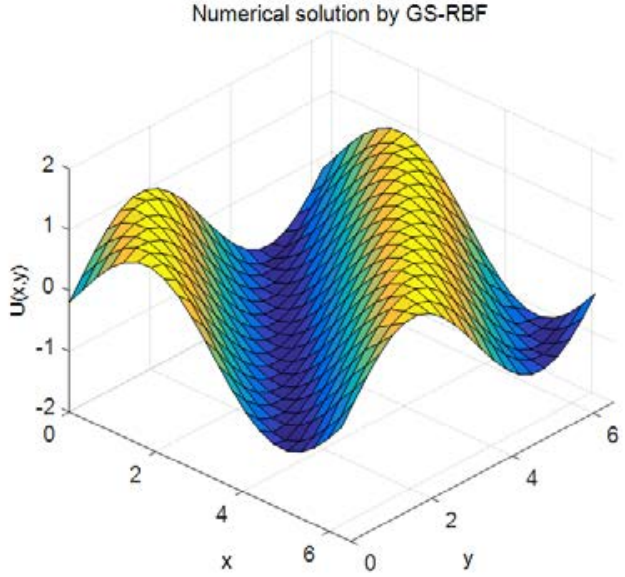

(a)

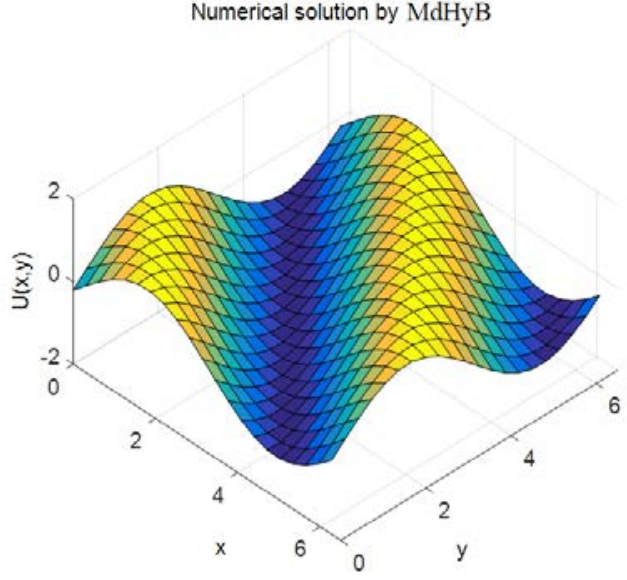

(b)

FIG. 5. Numerical solution measured along the straight line using 441 uniformlydistributed computation nodes; (above) at $t=1.0 \mathrm{~s}$, with $\Delta t=0.05$, and (below) at $t=$ $5.0 s$, with $\Delta t=0.1$. 


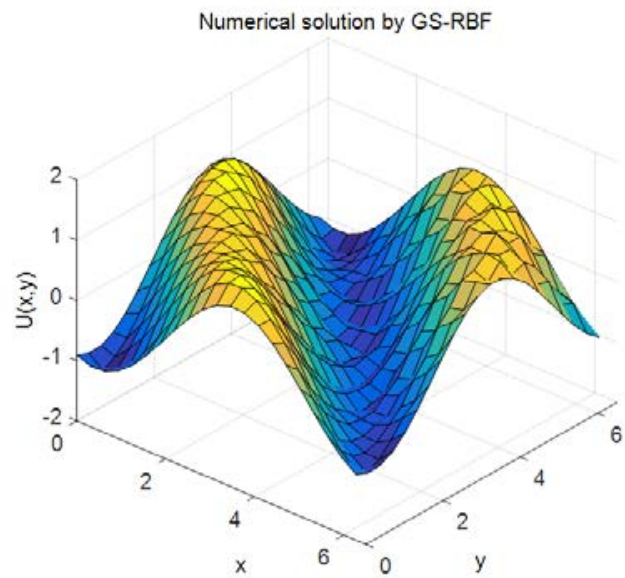

(a)

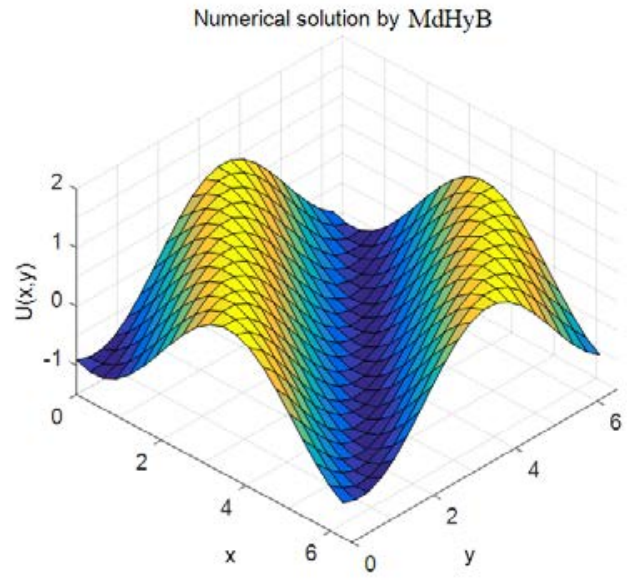

(b)

FIG. 6. Solution profile surface numerically computed at $t=1.0$, with $\Delta t=0.05$ using 441 uniformly-distributed computation nodes; (a) that obtained from Gaussian RBF (GS) $\left(\rho_{\text {best }}=12.25\right)$, and (b) obtained from using the proposed RBF(MdHyB).

The effect of time-increment to the final solution is demonstrated through Fig $4-6$. The comparison of solution on the straight line $x=y$ on the domain is plotted in Fig 4 at two values of time- $t$.It is clearly seen that at small $t^{\prime} s(t \leq 2.00)$, most RBFs are capable of reproducing good numerical solution for the problem, see Fig.4 (above) and Fig.5. However, when time increases all RBFs are seen to have lost their effectiveness with the burden of choosing most suitable shape are still apparent. Some promising figure is nevertheless discovered at $t=1.0$, with $\Delta t=0.05$ using 441 uniformly-distributed computation nodes where the proposed RBF is clearly seen to outperform the famous Gaussian RBF (GS) $\left(\rho_{\text {best }}=12.25\right)$.

\section{CONCLUSION}

The investigation begins with realizing the promising results obtained for scattered data interpolation with a combination of two RBF; Gaussian and Cubic types. The RBF is further applied in conjunction with the collocation meshfree method and some good results are revealed. Furthermore, the Hybrid-RBF is modified to the form where the effect of shape parameter in the Gaussian part is completely eliminated and this new form is being proposed in this research. Three examples, linear and nonlinear, are numerically solved by the collocation meshfree method in conjunction with the newly-proposed RBF. It is interestingly found that with the newly invented hybrid and parameter free RBF, the results quality is noticeably improved. Furthermore, while finding the most suitable shape parameter remains a great disadvantage for most forms of RBFs, this problem vanishes when using the modified RBF proposed in this work. This certainly makes it a promising alternative RBF and truly deserves further investigation. 


\section{ACKNOWLEDGEMENT}

The corresponding author would like to express his sincere appreciation to the Centre of Excellence in Mathematics, Thailand, for their kind support.

\section{REFERENCES}

[1] Kansa E. J., Multiquadrics-A scattered data approximation scheme with applications to computational fluid-dynamics-II solutions to parabolic, Computers Math. Applic, 1990, vol. 19, p. 147-161.

[2] Sarra S. A. and Kansa E. J., Multiquadric Radial Basis Function Approximation Methods for the Numerical Solution of Partial Differential Equations, Adv. Comput. Math, 2009.

[3] Yaghouti M. and Azarboni H. R., Determining optimal value of the shape parameter c in RBF for unequal distances topographical points by Cross-Validation algorithm, Journal of Mathematical Modeling, 2017, vol. 5, p. 53-60.

[4] Chanthawara K. and Kaennakham S., A Numerical Experiment on Optimal Inverse Multiquadric RBF Shape Parameter in the Dual Reciprocity Boundary Element Method for Convective-Dominated Problems, The 11th IMT-GT International Conference on Mathematics, Statistics and Its Applications 2015(ICMSA 2015), 2016, p. 119-131

[5] Kaennakham S., Chanthawara K. and Toutip W., Optimal Radial Basis Function (RBF) for Dual Reciprocity Boundary Element Method (DRBEM) applied to Coupled Burgers' Equations with Increasing Reynolds Number, Australian Journal of Basic and Applied Sciences, 2014, p. 462-476.

[6] Wang J. G. and Liu G. R., On the optimal shape parameters of radial basis functions used for 2-D meshless methods, Computer Methods in Applied Mechanics and Engineering, Boca Raton, 2002, vol. 191, p. 2611-2630.

[7] Chuathong N., Kaennakham S. and Toutip W., Numerical solutions of 2D nonlinear PDEs using Kansa's meshless method and the search for optimal radial basis function, Proceeding of 19th International Annual Symposium on Computational Science and Engineering, Ubon Ratchthani university, Ubon Ratchathani, Thailand, 2015, p. 13-15

[8] Mishra P. K., Nath S. K., Sen M. K. and Fasshauer G. E., Hybrid Gaussian-cubic radial basis functions for scattered data interpolation, Computational Geosciences, 2018, p. 1203-1218.

[9] Kaennakham S. and Chuathong N., Numerical simulation of convection-diffusion phenomena by four inverse-quadratic-RBF domain-meshfree schemes, International Journal of Multiphysics, 2019, vol. 13, p. 1-30.

[10] Kaennakham S. and Chuathong N., Solution to a Convection-Diffusion Problem Using a New Variable Inverse-Multiquadric Parameter in a Collocation Meshfree Scheme, International Journal of Multiphysics, 2017, vol. 11, p. 359-374.

[11] Kaennakham S. and Chuathong N., Numerical Simulation of Convection-Diffusion Phenomena by Four Inverse-Quadratic-RBF Domain-Meshfree Schemes, International Journal of Multiphysics, 2019, vol. 13, p. 1-30.

[12] Chuathong N., An Automatic Centroid-Node Adaptive Meshless (CNAM) Method For Solving Convection-Diffusion Problems, Khon Kaen University, Thailand, 2016. 
[13] Chen C. S., Kuhn G., Li J. and Mishuris G., Radial basis functions for solving near singular Poisson problems, Communications in Numerical Methods in Engineering, 2003, p. 333-347. John Wiley \& Sons, Ltd.

[14] Dehghan M. and Tatari M., The radial basis functions method for identifying an unknown parameter in a parabolic equation with overspecified data, Numerical Methods for Partial Differential Equations, 2007, vol. 23, p. 984 - 997. Wiley Online Library

[15] Fasshauer G. E., Newton iteration with multiquadrics for the solution of nonlinear PDEs, Computers \& Mathematics with Applications, 2002, vol. 43, p. 423-438. Elsevier

[16] Linesawat K., The dual reciprocity boundary element method for nonlinear problems using compactly supported radial basis function, Khon Kaen University, Thailand, 2010.

[17] Pao C. V., Block monotone iterative methods for numerical solutions of nonlinear elliptic equations, Numer. Math., 1995, vol. 72, p. 239-262. 